Pacific Journal of Mathematics

IMAGES AND PRE-IMAGES OF LOCALIZATION MAPS

Herbert Copeland, Jr. AND ALbert Oscar Shat 


\title{
IMAGES AND PRE-IMAGES OF LOCALIZATION MAPS
}

\author{
Arthur H. Copeland, JR. AND Albert O. Shar
}

This paper treats the induced function $\mathscr{L}_{*:}[X, Y] \rightarrow\left[X, Y_{P}\right]$ when $X$ and $Y$ are spaces having "just enough" structure and $\mathscr{L}: Y \rightarrow Y_{P}$ is a localization map. Special attention is paid to the images of finite subsets of $[X, Y]$ and the preimages of finite subsets of $\left[X, Y_{P}\right]$. While our results are not restricted to finite $[X, Y]$ we do, as an immediate corollary of our main theorem, establish necessary and sufficient conditions for the finiteness of $\left[X, Y_{P}\right]$.

1. Introduction. If $Y$ is a homotopy-associative $H$-space ${ }^{1}$ it is relatively easy to study properties of $\mathscr{L}_{*}:[X, Y] \rightarrow\left[X, Y_{P}\right]$ where $P$ is a set of prime integers and $\mathscr{L}: Y \rightarrow Y_{P}$ is a localization map [1], $|7|$. The most obvious technique to use is to note that $[X, Y]$ and $\left[X, Y_{P}\right]$ are nilpotent groups, $\mathscr{L}_{*}$ is a homomorphism and $\left[X, Y_{P}\right]$ is isomorphic to $[X, Y]_{P}$, the localization of the group $[X, Y]$. For example if $Y$ is a homotopy associative $H$-space of type $\left(n_{1}, \cdots, n_{r}\right)$ and $X$ is finite $C W$, it is immediate that $[X, Y]$ is finite if, and only if $\bigoplus_{i=1}^{r} H^{n}(X ; Q)=0$. Even without finiteness restrictions there are numerous results which can be based on the structure of finitely generated nilpotent groups [2].

At the other exteme, there is in general little that one can say about the system $\mathscr{L}_{*}:[X, Y] \rightarrow\left[X, Y_{P}\right]$ if there are no restrictions on $Y$. A notable exception to this statement are the results of Hilton, Mislin and Roitberg [3].

In the present paper, while some of our results are general and overlap [3], we are primarily concerned with spaces $Y$ which have "just enough structure." This class of spaces is essentially the $p$ universal spaces of [6], and in particular contains all rational $H$-spaces and co- $H$-spaces (that is, spaces whose localization at the empty set is an $H$-space or co-H-space). While our results are not restricted to finite $[X, Y]$ we do, as an immediate corollary of our main theorem, prove:

Let $Y$ be a finitely generated rational $H$-space or co-H-space, let $X$ be a finite $C W$-space and let $\mathscr{L}: Y_{P} \rightarrow Y_{S}$ be a localization map $(P \supset S$ are subsets, not necessarily proper, of the set of prime integers). Then $\left[X, Y_{P}\right]$ is finite if, and only if $\left[X, Y_{S}\right]$ is finite. Furthermore, $\mathscr{L}_{*}:\left[X ; Y_{P}\right] \rightarrow\left[X ; Y_{S}\right]$ is epic.

${ }^{1}$ Unless otherwise noted all spaces are assumed to be of finite type and simple, or the $P$-localizations of such spaces. 
The paper is divided as follows. Section 2 contains the notation definitions, and basic results we need. The third section is devoted to proving an algebraic result concerning the relationship between homotopy-principal fibrations and localizations. In $\S 4$ we prove the basic theorem of this paper. The final section is devoted to corollaries and applications of this theorem.

2. Notation. If $P$ is a set of prime numbers, let $P^{\prime}$ denote the complementary set of primes, and $\langle P\rangle$ the multiplicative set of integers generated by $P$ (i.e., all products of powers of members of $P$ ). Let $Z$ be the group (or ring) of integers, $Q$ the rational numbers, and $Z_{P}$ the rational numbers with denominators in $\left\langle P^{\prime}\right\rangle$. If $n$ is an integer, let $Z / n$ denote the group of integers modulo $n$. An integer is said to be prime to $P$ if it is prime to each member of $P$, and a finite group is prime to $P$ if its order is prime to $P$.

A map is a continuous, pointed function between pointed topological spaces. We do not make a notational distinction between maps and their homotopy classes. As usual, $[X, Y]$ denotes the set of homotopy classes of maps $X \rightarrow Y$.

An abelian group $G$ is called $P$-local if $G$ is isomorphic to $G \otimes Z_{P}$. The reader is referred to [2] for the extension of this notion to nilpotent groups. A pointed space is called $P$-local if its homotopy groups (hence its homology groups) are $P$-local. A map $\mathscr{L}: X \rightarrow Y_{P}$ is called a localization map if

(1) $X_{P}$ is a $P$-local pointed space, and

(2) For each map $f$ of $X$ into a $P$-local space $Y$, there is a unique map $g: X_{P} \rightarrow Y$ with $f=g \circ \mathscr{L}$.

The reader is referred to [7], [1] and [5] for more complete discussions of this topic. In particular, every simple, pointed $C W$-space $X$ admits a localization map $\mathscr{L}: X \rightarrow X_{P}$ with $X_{P}$ a pointed, simple $C W$-space. The space $X_{P}$ is unique up to homotopy equivalences and is called the localization of $X$. It is immediate that, for spaces $X$ and $Y$ admitting localizations, a map $h: X \rightarrow Y$ induces a map $h_{P}: X_{P} \rightarrow Y_{P}$ that commutes with the given localization maps. The map $h$ is a $P$-equivalence provided $h_{P}$ is a weak homotopy equivalence.

A simple, pointed $C W$-space $Y$ is called $P$-universal if, for each finite abelian group $G$ prime to $P$ and for each $N>0$, there is a $P$-equivalence $h$ on $Y$ such that $\pi_{n}(h) \otimes 1$ is trivial on $\pi_{n}(Y) \otimes C$ for all $n \leqq N$. It is known that $H$-spaces and co- $H$-spaces are $P$ universal for all $P$ [6]. Furthermore, if $Y$ is $P$-universal and $\left\{Y_{n}\right\}$ is a Postnikov system for $Y$, then each $Y_{n}$ is $P$-universal and the induced maps $h_{n}$ are compatible with the system [4].

Two path-connected, pointed $C W$-spaces $X$ and $Y$ form a finite obstruction pair if the groups 


$$
H^{n+i}\left(X ; \pi_{n}(Y)\right) \quad i=0,1
$$

are finitely generated for all $n$ and are trivial for large $n$. The least integer $N$ such that $H^{n+i}\left(X ; \pi_{n}(Y)\right)=0$ for $n>N$ and $i=0,1$ is called the dimension of the pair.

THEOREM 2.1. If $X, Y$ is a finite obstruction pair and if $Y$ is a homotopy-associative $H$-space, then $[X, Y]$ is a finitely generated nilpotent group.

Proof. The proof is by induction on the index $n$ of the Postnikov complex $Y_{n}$ of $Y$. The result is trivial for the one-point space $Y_{0}$. Suppose $\left[X ; Y_{n-1}\right]$ is finitely generated and nilpotent. Let $p: Y_{n} \rightarrow Y_{n-1}$ be the fibering induced by the Postnikov invariant $k: Y_{n-1} \rightarrow K\left(\pi_{n}, n+1\right)$ and let $i: K\left(\pi_{n}, n\right) \rightarrow Y_{n}$ be the inclusion map of the fibre into the total space. Since $i$ and $p$ are multiplicative maps of $H$-spaces, the sequence,

$$
\left[X ; K\left(\pi_{n}, n\right)\right] \stackrel{i_{*}}{\longrightarrow}\left[X ; Y_{n}\right] \stackrel{p_{*}}{\longrightarrow}\left[X ; Y_{n-1}\right]
$$

is an exact sequence of groups and homomorphisms. Note that

$$
\left[X ; K\left(\pi_{n}, n\right)\right]=H^{n}\left(X ; \pi_{n}(Y)\right)
$$

is a finitely generated abelian group. Thus $\left[X ; Y_{n}\right]$ is a central extension of the finitely generated abelian group $i m\left(i_{*}\right)$ by a finitely generated nilpotent group $i m\left(p_{*}\right)$, and hence is finitely generated and nilpotent. Since $\left[X ; K\left(\pi_{n}, n\right)\right]$ and $\left[X ; K\left(\pi_{n}, n+1\right)\right]$ are eventually trivial, $[X ; Y]=\left[X ; Y_{n}\right]$ for $n$ sufficiently large, whence the result follows. It should be noted that this result is a generalization of a well known result of G. W. Whitehead [8].

3. Principal fibrations and localizations. Let $(E, F)$ be a pair of path-connected pointed spaces with $F \subset E$. A map $\phi: F \times E \rightarrow E$ is called a principal action provided:

(1) There is a homotopy-associative $H$-structure $\mu: F \times F \rightarrow F$ whose composite with the inclusion $F \subset E$ is homotopic to $\phi \mid F \times F$, and

(2) The diagram

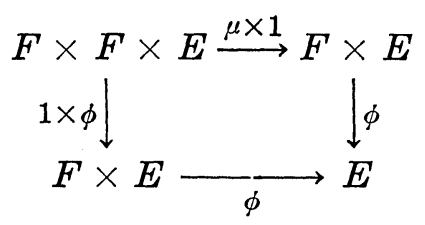

is homotopy-commutative. 
This situation arises when $F$ is the space of loops on some space $C$ and $E$ is the total space of a fibering $p: E \rightarrow B$ induced by a map $B \rightarrow C$. Our applications are to such fiberings.

Suppose $P$ is a collection of primes. Let $\mathscr{L}: E \rightarrow E_{P}$ and $\mathscr{L}^{\prime}: F \rightarrow F_{P}$ be localization maps. Then there is no loss of generality in regarding $F_{P}$ as a subspace of $E_{P}$ and $\mathscr{L}^{\prime}$ as a restriction of $\mathscr{L}$. Recall that $(F \times E)_{P}$ has the homotopy type of $F_{P} \times E_{P}$. [1, p. 34], [7], [5].

LEMMA 3.1. The localization $\phi_{P}: F_{P} \times E_{P} \rightarrow E_{P}$ is a principal action.

The proof is a sequence of routine applications of the universality of localization maps.

Let $X$ be a $C W$-space. If $f \in[X, E]$ and $\tau \in[X, F]$, let $\tau_{*} f \in[X, E]$ be the homotopy class, $\tau_{*} f=\phi_{*}(\tau \times f)$. Define

$$
K_{f}=\left\{\tau \in[X, F]\left[\tau_{*} f=f\right\} \text { and }[X, E]_{f}=\left\{\tau_{*} f \mid \tau \in[X, F]\right\} .\right.
$$

It is immediate that $K_{f}$ is a subgroup of the group $[X, F]$ and that the map $\Phi:[X, F] \rightarrow[X, E]_{f}$ defined by $\Phi(\tau)=\tau_{*} f$ is surjective.

Lemma 3.2. If $K_{f}$ is normal in $[X, F]$, then $[X, E]_{f}$ has a group structure; it is the quotient of $[X, F]$ by $K_{f}$.

Proof. Define the product on $[X, E]_{f}$ by $\left(\tau_{1 *} f\right)\left(\tau_{2 *} f\right)=\left(\tau_{1} \tau_{2}\right)_{*} f$. This is well-defined since $K_{f}$ is normal and the action is associative. One easily verifies that $\Phi$ is a homomorphism whose kernel is $K_{f}$.

A morphism of principal actions is defined to be a map $g:(E, F) \rightarrow$ $\left(E^{\prime}, F^{\prime}\right)$ such that $\phi^{\prime} \circ((g \mid F) \times g)$ is homotopic to $g \circ \phi$. Note that this is equivalent to requiring the diagram,

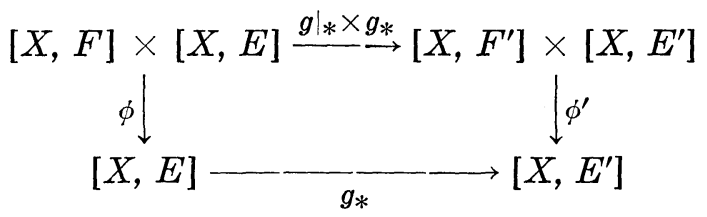

to commute for all spaces $X$. If $K_{f}$ is normal in $[X, F]$ and $K_{g_{*} f}$ is normal in $\left[X, F^{\prime}\right]$, then it follows easily from Lemma 3.2 that $g$ induces a homomorphism $g_{*}:[X, E]_{f} \rightarrow\left[X, E^{\prime}\right]_{g_{*} f}$. In particular, a localization map $\mathscr{L}:(E, F) \rightarrow\left(E_{p} F_{P}\right)$ induces such a homomorphism.

Theorem 3.3. Suppose $\phi: F \times E \rightarrow E$ is a principal action and that $X$ is a $C W$-space such that $X, F$ and $X, E$ are finite obstruc- 
tion pairs. If $K_{f}$ is normal, then a localization $\mathscr{L}:(E, F) \rightarrow\left(E_{P}, F_{P}\right)$ induces an isomorphism of the local group $\left([X, E]_{f}\right)_{P}$ onto $\left[X, E_{P}\right]_{\mathscr{X}_{*} f}$ and

$$
\operatorname{ker}\left(\mathscr{L}_{*}:[X, E]_{f} \longrightarrow\left[X, E_{P}\right]_{\mathscr{L}_{* f}}\right)
$$

is finite of order prime to $P$.

Proof. Since $F$ is a homotopy-associative $H$-space and $X, F$ is a finite obstruction pair, $[X, F]$ is a finitely generated nilpotent group. Thus $[X, F]_{P}$ is naturally isomorphic to $\left[X, F_{P}\right]$. It then follows that $K_{f}$ is finitely generated and nilpotent, and that $\left(K_{f}\right)_{P}$ is isomorphic to $K_{\mathscr{L} * f}$, whence $[K, E]_{f}$ is finitely generated nilpotent, and $\left([X, E]_{f}\right)_{P}$ is isomorphic to $\left[X, E_{P}\right]_{\mathscr{C}^{*} f}$, whence $\operatorname{ker} \mathscr{L}_{*}$ is finitely generated, nilpotent and every element has finite order. It follows at once that ker $\mathscr{L}_{*}$ is finite. A result of Hilton [2, p. 266] shows that each element has order prime to $P$.

4. The main theorem.

TheOREM 4.1. Let $P$ be a set of primes. Suppose $X, Y$ is a finite obstruction pair with $Y$ P-universal. If $\left\{g_{\alpha} \mid \alpha \in A\right\} \subset\left[X, Y_{P}\right]$ is a finite set of homotopy classes, then there exist a localization map $L: Y \rightarrow Y_{P}$ and classes $\widetilde{g}_{\alpha} \in[X, Y]$ such that $L_{*}\left(\widetilde{g}_{\alpha}\right)=g_{\alpha}$. Furthermore, for any localization map $\mathscr{L}: Y \rightarrow Y_{P}$, the sets $\mathscr{L}_{*}^{-1}\left(g_{\alpha}\right)$ are finite.

Proof. The proof is by induction on the dimension of the finite obstruction pair $X, Y$. Note that if the dimension is $n$, there is no loss in replacing $Y$ and $Y_{P}$ by their Postnikov complexes $Y_{n}$ and $\left(Y_{P}\right)_{n}$, respectively. Since $Y_{0}=\left(Y_{P}\right)_{0}$ is a single point, the initial step is trivial.

Suppose the result has been established for pairs $X, Y$ of dimen-

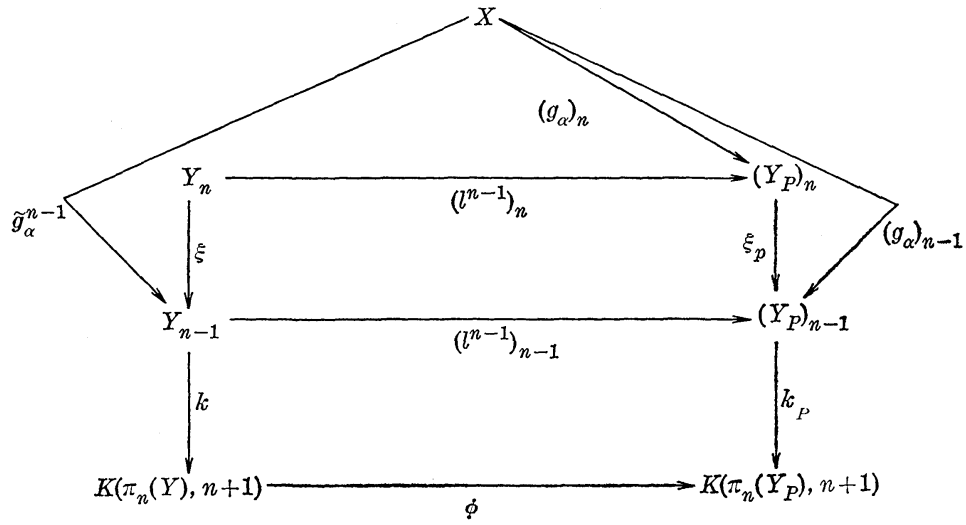


sion $\leqq n-1$. In particular, given a pair $X, Y$ of dimension $n$, assume we have maps $\widetilde{g}_{\alpha}^{n-1}: X \rightarrow Y_{n-1}$ and a localization map $\mathscr{L}^{n-1}: Y \rightarrow Y_{P}$ such that $\left(\mathscr{L}^{n-1}\right)_{n-1} \widetilde{g}_{\alpha}^{n-1}=\left(g_{\alpha}\right)_{n-1}$. These fit into the homotopycommutative diagram above where $k, k_{P}$ are maps corresponding to Postnikov invariants and $\phi=K\left(\pi_{n}\left(\mathscr{L}^{n-1}\right), n+1\right)$ is a map between Eilenberg-MacLane spaces corresponding to the homomorphism $\pi_{n}^{\left(\mathscr{S}^{n-1}\right)}: \pi_{n}(Y) \rightarrow \pi_{n}\left(Y_{P}\right)$. The existence of maps $\left(g_{\alpha}\right)_{n-1},\left(g_{\alpha}\right)_{n},\left(\mathscr{L}^{n-1}\right)_{n-1}$ and $\left(\mathscr{L}^{n-1}\right)_{n}$ induced by $g_{\alpha}$ and $\mathscr{L}^{n-1}$ and making the diagram homotopy-commutative is insured by the construction of localization [7].

Since $\left(g_{\alpha}\right)_{n-1}$ has a lifting $g_{\alpha}$, it holds that $k_{P}\left(g_{\alpha}\right)_{n-1}=\phi k \widetilde{g}_{\alpha}^{n-1}$ is trivial for each $\alpha$. Thus there is an integer $m_{\alpha}$ prime to $P$ such that

$$
m_{\alpha}\left(k \widetilde{g}_{\alpha}^{n-1}\right) \in\left[X ; K\left(\pi_{n}(Y), n+1\right)\right]=H^{n+1}\left(X ; \pi_{n}(Y)\right)
$$

is trivial. Let $m$ be the product $m=\Pi\left\{m_{\alpha} \mid \alpha \in A\right\}$. Since $Y$ is $P$ universal, there is a $P$-equivalence $h$ on $Y$ such that $\pi_{n}(h) \otimes 1$ is trivial on $\pi_{n}(Y) \otimes(Z / m)$. Note that $k h_{n-1} \widetilde{g}_{\alpha}^{n-1}$ is trivial for each $\alpha$, whence $h_{n-1} \widetilde{g}_{\alpha}^{n-1}$ has a lifting $f_{\alpha}: X \rightarrow Y_{n}$. Since $h$ is a $P$-equivalence, the induced map $h_{P}$ on $Y_{P}$ is a homotopy equivalence. Let $h_{P}^{-1}$ be a homotopy inverse and set $\mathscr{L}^{\prime}=h_{P}^{-1} \mathscr{L}^{n-1}$. Then,

$$
\xi_{P}\left(\mathscr{L}^{\prime}\right)_{n} f_{\alpha}=\left(h_{P}^{-1}\right)_{n-1}\left(\mathscr{L}^{n-1}\right)_{n-1} h_{n-1} \widetilde{g}_{\alpha}^{n-1}=\xi_{P}\left(g_{\alpha}\right)_{n} .
$$

Since $\xi_{P}$ is a principal fibration with fibre $K\left(\pi_{n}\left(Y_{P}\right), n\right)$, there is a $\operatorname{map} \tau_{\alpha}: X \rightarrow K\left(\pi_{n}\left(Y_{P}\right), n\right)$ such that

$$
\tau_{\alpha} *\left(\left(\mathscr{L}^{\prime}\right)_{n} f_{\alpha}\right)=\left(g_{\alpha}\right)_{n},
$$

where the asterisk denotes the action of the fibre on the total space $\left(Y_{P}\right)_{n}$. But there exists an integer $q_{\alpha}$ prime to $P$ and a map $\tau_{\alpha}^{\prime}: X \rightarrow$ $K\left(\pi_{n}(Y), n\right)$ such that the homotopy class $\tau_{\alpha}^{\prime}$ maps onto $q_{\alpha} \tau_{\alpha}$ under the localization map,

$$
\theta=K\left(\pi_{n}\left(\mathscr{L}^{\prime}\right), n\right): K\left(\pi_{n}(Y), n\right) \longrightarrow K\left(\pi_{n}\left(Y_{P}\right), n\right) .
$$

Let $q=\Pi\left\{q_{\alpha} \mid \alpha \in A\right\}$ and let $H$ be a $P$-equivalence on $Y$ such that $\pi_{n}(H) \otimes 1$ is trivial on $\pi_{n}(Y) \otimes(Z / q)$. Thus each element in the image of $\pi_{n}(H)$ is divisible by $q$, and the induced map $H_{*}$ on $\left[X ; K\left(\pi_{n}(Y), n\right)\right]=H^{n}\left(X ; \pi_{n}(Y)\right)$ has the same property. For each $\alpha$, let $\tau_{\alpha}^{\prime \prime} \in\left[X ; K\left(\pi_{n}(Y), n\right)\right]$ be an element such that $q \tau_{\alpha}^{\prime \prime}=H_{*}\left(\tau_{\alpha}^{\prime}\right)$. Note that $q / q_{\alpha}$ is an integer, and let $\tilde{\tau}_{\alpha}=\left(q / q_{\alpha}\right) \tau_{\alpha}^{\prime \prime}$. As before, $H$ induces a homotopy equivalence $H_{P}$ on $Y_{P}$ and we select a homotopy inverse $H_{P}^{-1}$. Let $L=H_{P}^{-1} \mathscr{L}^{\prime}$ and set $\widetilde{g}_{\alpha}^{n}=\tilde{\tau}_{\alpha}{ }^{*}\left(H_{n} f_{\alpha}\right)$. If we replace $Y_{n}$ by $Y$ we obtain

$$
L \widetilde{g}_{\alpha}=H_{P}^{-1} \mathscr{L}^{\prime}\left(\widetilde{\tau}_{\alpha}^{*}\left(H f_{\alpha}\right)\right)=H_{P}^{-1}\left(\left(\theta \widetilde{\tau}_{\alpha}\right)^{*}\left(H_{P} \mathscr{L}^{\prime} f_{\alpha}\right)\right)
$$

Now, 


$$
\left(H_{P}^{-1}\right)_{*} \theta \widetilde{\tau}_{\alpha}=\left(H_{P}^{-1}\right)_{*}\left(q / q_{\alpha}\right) \theta_{*}\left(\tau_{\alpha}^{\prime \prime}\right)=\left(1 / q_{\alpha}\right)\left(H_{P}^{-1}\right)_{*} \theta_{*} H_{*} \tau_{\alpha}^{\prime}=\tau_{\alpha},
$$

whence,

$$
L \widetilde{g}_{\alpha}=\tau_{\alpha *}\left(H_{P}^{-1} H_{P} \mathscr{L}^{\prime} f_{\alpha}\right)=g_{\alpha} .
$$

This establishes the existence of a localization $L$ such that $L_{*}^{-1}\left(g_{\alpha}\right)$ is nonempty.

Let $\mathscr{L}: Y_{n} \rightarrow\left(Y_{P}\right)_{n}$ be any localization and let $\alpha \in A$. By the inductive hypothesis, we know that $\xi_{*}\left(\mathscr{L}_{*}^{-1}\left(g_{\alpha}\right) n\right)=\left(\mathscr{L}_{n-1}\right)_{*}^{-1}\left(g_{\alpha}\right)_{n-1}$ is a finite set. But if $f, f^{\prime} \in \mathscr{L}_{*}^{-1}\left(g_{\alpha}\right)_{n}$ and $\xi_{*} f=\xi_{*} f^{\prime}$, then there exists a class $\tau \in\left[X ; K\left(\pi_{n}(Y), n\right)\right]$ such that $\tau_{*} f^{\prime}=f$. Thus by (3.3) $f^{\prime}$ lies in the finite set $\operatorname{ker}\left(\mathscr{L}_{*}:\left[X, Y_{n}\right]_{f} \rightarrow\left[X,\left(Y_{P}\right)_{n}\right]_{\left(g_{\alpha}\right) n}\right)$ and $\mathscr{L}_{*}^{-1}\left(g_{\alpha}\right)_{n}$ is finite.

REMARKS. It should be noted that the commutativity of maps between Postnikov decompositions is critical at two points in the above proof. This may be seen by the fact that it is necessary to replace the initial localization map by a different one in two steps and each time require that the diagrams commute so that we may continue the induction process.

We also remark that $([5], 5.3$, p. 607) contains a simply-connected version of the first part of Theorem 4.1. We would also like to note that the proof of 4.1 goes over, mutatis mutandis, to the more general class of nilpotent spaces.

Futhermore note that given $\left\{g_{\alpha} \mid \alpha \in A\right\}$ a finite subset of $\left[X, Y_{P}\right]$ the $P$-universality of $Y$ was only used to assure the existence of a localization map $L: Y \rightarrow Y_{P}$ and of classes $\left\{\widetilde{g}_{\alpha}\right\} \in[X, Y]$ with $L_{*}\left(\widetilde{g}_{\alpha}\right)=$ $g_{\alpha}$. Thus we get:

CoROllaRy 4.2. Let $\mathscr{L}: Y \rightarrow Y_{P}$ be any localization map with $X, Y$ a finite obstruction pair. If $\left\{g_{\alpha} \mid \alpha \in A\right\} \subseteq\left[X, Y_{P}\right]$ is finite then $\mathscr{L}_{*}^{-1}\left(\left\{g_{\alpha} \mid \alpha \in A\right\}\right)$ is finite.

This is essentially the same statement as [3, Corollary 2.2].

5. Applications. If $Y$ is $P$-universal, then $Y_{Q}$ is $P$-universal for all sets of primes $Q \supset P$. Further, $X$ finite and $Y$ having finitely generated homotopy in each dimension implies that $X, Y$ is a finite obstruction pair. Therefore as an immediate corollary of 4.1 we have:

THEOREM 5.1. Let $X$ be a finite, pointed $C W$-space. Let $Y$ be $P$-universal and have finitely generated homotopy in each dimension. If $\left\{g_{\alpha} \mid \alpha \in A\right\}$ is a finite subset of $\left[X, Y_{P}\right]$, then there exists a localization map $L: Y \rightarrow Y_{P}$ and homotopy classes $\widetilde{g}_{\alpha} \in[X, Y]$ such that 
$L_{*}\left(\widetilde{g}_{\alpha}\right)=g_{\alpha} . \quad$ Further, for any localization map $\mathscr{L}: Y \rightarrow Y_{P}$, the sets $\mathscr{L}^{-1}\left(g_{\alpha}\right)$ are finite.

If $X, Y$ is a finite obstruction pair and $Q$ is a set of primes then $X_{Q}, Y_{Q}$ is not necessarily a finite obstruction pair. However the proof of 4.1 extends immediately to give

THEOREM 5.2 Let $X$ be a finite, pointed $C W$-space. Let $Y$ be $P$-universal for some set of primes $P$ and have finitely generated homotopy in each dimension. Let $Q$ be a set of primes containing $P$. If $\left\{g_{\alpha} \mid \alpha \in A\right\}$ is a finite subset of $\left[X, Y_{P}\right]$, then there exists a localization map $L: Y_{Q} \rightarrow Y_{P}$ and homotopy classes $\widetilde{g}_{\alpha} \in\left[X, Y_{Q}\right]$ such that $L_{*}\left(\widetilde{g}_{\alpha}\right)=g_{\alpha}$. Further, for any localization map $\mathscr{L}: Y_{Q} \rightarrow Y_{P}$ the sets $\mathscr{L}_{*}^{-1}\left(g_{\alpha}\right)$ are finite.

THEOREM 5.3. Let $P$ be a set of primes and $X, Y$ a finite obstruction pair with $Y$ P-universal. Let $\mathscr{L}: Y \rightarrow Y_{P}$ be any localization. Then $[X, Y]$ is finite if, and only if, $\left[X, X_{P}\right]$ is finite. Furthermore, $\mathscr{L}_{*}:[X, Y] \rightarrow\left[X, Y_{P}\right]$ is epic.

Proof. First assume $[X, Y]$ is finite of cardinality $r$. If $\left[X, Y_{P}\right]$ is not finite, let $g_{1} \cdots, g_{r+1}$ be $r+1$ distinct homotopy classes in $\left[X, Y_{P}\right] . \quad$ By 4.1 there exist $\widetilde{g}_{i} \in[X, Y]$ and a localization map $L: Y \rightarrow$ $Y_{P}$ with $L_{*}\left(\widetilde{g}_{i}\right)=g_{i}$. But this implies that the cardinality of $[X, Y]$ is greater than $r$, a contradiction. Thus $\left[X, Y_{P}\right]$ is finite. If $\left[X, Y_{P}\right]$ is finite, then again by 4.1 , there exists a localization map $L: Y \rightarrow Y_{P}$ which is epic. Furthermore, $L_{*}^{-1}(g)$ is finite for each $g \in\left[X, Y_{P}\right]$. Thus $[X, Y]$ is finite.

Finally, let $\mathscr{L}: Y \rightarrow Y_{P}$ be a localization map. Since any two localization maps differ by a homotopy equivalence, we have $\mathscr{L}=$ $L \circ h$, where $L_{*}$ is epic and $h_{*}$ is an isomorphism of sets. Therefore, $\mathscr{L}_{*}:[X, Y] \rightarrow\left[X, Y_{P}\right]$ is epic.

Corollary 5.4. Let $X, Y$ be a finite obstruction pair. Then for any set of primes $P$, the set $[X, Y]$ is finite if $\left[X, Y_{P}\right]$ is finite.

Proof. Immediate by 4.2 .

The example, $X=Y=S^{n}$ and $P$ any set of primes (except the set of all primes), shows that a localization need not induce a surjection when $[X, Y]$ is infinite.

As noted before, any $H$-space or co- $H$-space is $P$-universal for all $P$ [6]. Therefore we get: 
Corollary 5.5. Let $X$ be a finite, pointed $C W$-space and let $Y$ be an $H$-space or co-H-space whose homotopy is finitely generated in each dimension. Then for any set $P$ of primes and any localization map $\mathscr{L}: Y \rightarrow Y_{P},[X, Y]$ is finite if, and only if, $\left[X, Y_{P}\right]$ is finite. Furthermore, $\mathscr{L}_{*}:[X, Y] \rightarrow\left[X, Y_{P}\right]$ is epic.

In particular, if $Y$ is a finite $C W H$-space of type $\left(n_{1}, \cdots, n_{r}\right)$, then the number of homotopy classes of multiplications on $Y$ is the cardinality of $[Y \wedge Y, Y]$. Since $Y_{0} \cong \Pi_{i=1}^{r} K\left(Q, n_{i}\right)$ we have $[Y \wedge Y, Y]=\bigoplus_{i=1}^{r} H^{n_{i}}(Y \wedge Y ; Q)$. Finally, since 0 is the only finite $Q$-module, we get:

Corollary 5.6. Let $Y$ be a finite $C W H$-space of type $\left(n_{i}, \cdots, n_{r}\right)$. $Y$ has finitely many homotopy classes of multiplications if, and only if, $H^{n_{i}}(Y \wedge Y ; Q)=0$ for $i=1, \cdots, r$.

We close this paper with the following conjectures.

Conjecture 5.7. $[X, Y]$ is finite if and only if $\left[X, Y_{P}\right]$ is finite.

Note that in the light of 4.2 this is equivalent to the statement that $\left[X, Y_{0}\right]$ is finite if $[X, Y]$ is finite and is therefore a question about the rationalization of the spaces $Y$.

Conjecture 5.8. If $Y$ is a rational space (i.e., $H_{*}(Y ; Z)=$ $\left.H_{*}(Y ; Q)\right)$ then $[X, Y]$ is either infinite or a single element.

If $Y$ is an $H$-space then $Y \simeq \Pi_{i \geqq 1} K\left(Q^{n_{i}}, i\right)$ and hence has a multiplication such that $[X, Y]$ is isomorphic to a $Q$ module. Thus 5.8 holds for $H$-spaces

Theorem 3.3 when applied to the proof of 4.1 shows that at every stage of the Postnikov system the indeterminacy in the choice of the lifting of a map $X \rightarrow Y_{n-1}$ to $X \rightarrow Y_{n}$ is finite of order prime to $P$ this suggests:

Conjecture 5.9. Let $Y$ be $P$-universal and $[X, Y]$ finite. Then there exists an integer $m$ such that $\sharp[X, Y]=m\left(\sharp\left[X, Y_{P}\right]\right)$ and $m$ is prime to $P$.

Combining 5.8 and 5.9 we could then prove,

Conjecture 5.10. Let $Y$ be $P$-universal and $[X, Y]$ finite. Then for each prime $p, \sharp\left[X, Y_{P}\right]$ is a power of $p$.

\section{REFERENCES}

1. A.K. Bousefield and D. Kan, Homotopy Limits, Completions and Localizations, Lecture notes in Math, v. 304, Springer-Verlag, Berlin and New York 1972. 
2. P. J. Hilton, Localization and cohomology of nilpotent groups, Math. Z., 132 (1973), 263-286.

3. P. Hilton, G. Mislin and J. Roitberg, Homotopical localization, Proc. London Math. Soc., (3) 26 (1973), 693-706.

4. D. W. Kahn, Induced Maps for Postnikov Systems, Trans. Amer. Math. Soc., 107 (1963), 423-450.

5. M. Mimura, G. Nishida and H. Toda, Localization of $C W$-complexes and its applications, J. Math. Soc. Japan, 23 (1971), 593-624.

6. M. Mimura, R. C. O'Neill and H. Toda, On P-equivalence in the sense of Serre, Japanese J. Math. 40 (1971), 1-10.

7. D. Sullivan, Geometric Topology, Part I, Mimeo Notes, M.I.T., 1970.

8. G. W. Whitehead, On mappings into group-like spaces, Comm. Math. Helv., 28 (1954), 320-328.

Received October 4, 1974 and in revised form February 7, 1975.

UNIVERSITY OF NEW HAMPSHIRE 


\section{PACIFIC JOURNAL OF MATHEMATICS}

\section{EDITORS}

RICHARD ARENS (Managing Editor)

University of California

Los Angeles, California 90024

\section{J. DugundJI}

Department of Mathematics University of Southern California Los Angeles, California 90007

D. Gilbarg and J. Milgram

Stanford University

Stanford, California 94305
University of Washington Seattle, Washington 98105

\section{ASSOCIATE EDITORS}
E. F. BECKENBACH
B. H. NeumanN
F. WolF
K. YoShIDA

\section{SUPPORTING INSTITUTIONS}

\author{
UNIVERSITY OF SOUTHERN CALIFORNIA \\ STANFORD UNIVERSITY \\ UNIVERSITY OF TOKYO \\ UNIVERSITY OF UTAH \\ WASHINGTON STATE UNIVERSITY \\ UNIVERSITY OF WASHINGTON \\ $\stackrel{*}{*} \stackrel{*}{*} \stackrel{*}{ }{ }^{*}$ AMERICAN MATHEMATICAL SOCIETY
}

The Supporting Institutions listed above contribute to the cost of publication of this Journal, but they are not owners or publishers and have no responsibility for its content or policies.

Mathematical papers intended for publication in the Pacific Journal of Mathematics should be in typed form or offset-reproduced, (not dittoed), double spaced with large margins. Underline Greek letters in red, German in green, and script in blue. The first paragraph or two must be capable of being used separately as a synopsis of the entire paper. Items of the bibliography should not be cited there unless absolutely necessary, in which case they must be identified by author and Journal, rather than by item number. Manuscripts, in triplicate, may be sent to any one of the editors. Please classify according to the scheme of Math. Reviews, Index to Vol. 39. All other communications should be addressed to the managing editor, or Elaine Barth, University of California, Los Angeles, California, 90024.

The Pacific Journal of Mathematics expects the author's institution to pay page charges, and reserves the right to delay publication for nonpayment of charges in case of financial emergency.

100 reprints are provided free for each article, only if page charges have been substantially paid. Additional copies may be obtained at cost in multiples of 50 .

The Pacific Journal of Mathematics is issued monthly as of January 1966. Regular subscription rate: $\$ 72.00$ a year (6 Vols., 12 issues). Special rate: $\$ 36.00$ a year to individual members of supporting institutions.

Subscriptions, orders for back numbers, and changes of address should be sent to Pacific Journal of Mathematics, 103 Highland Boulevard, Berkeley, California, 94708.

\section{PUBLISHED BY PACIFIC JOURNAL OF MATHEMATICS, A NON-PROFIT CORPORATION}

Printed at Kokusai Bunken Insatsusha (International Academic Printing Co., Ltd.), 270, 3-chome Totsuka-cho, Shinjuku-ku, Tokyo 160, Japan.

\section{Copyright (C) 1975 by Pacific Journal of Mathematics} Manufactured and first issued in Japan 


\section{Pacific Journal of Mathematics}

\section{Vol. 57, No. $2 \quad$ February, 1975}

Norman Larrabee Alling, On Cauchy's theorem for real algebraic curves with boundary .......

Daniel D. Anderson, A remark on the lattice of ideals of a Prüfer domain ..................

Dennis Neal Barr and Peter D. Miletta, A necessary and sufficient condition for uniqueness of

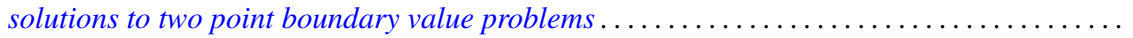

Ladislav Beran, On solvability of generalized orthomodular lattices . . . . . . . . . . ........

L. Carlitz, A three-term relation for some sums related to Dedekind sums . . . . . . . . . .....

Arthur Herbert Copeland, Jr. and Albert Oscar Shar, Images and pre-images of localization

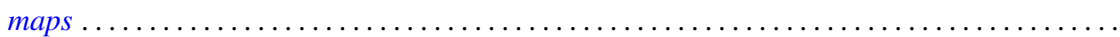

G. G. Dandapat, John L. Hunsucker and Carl Pomerance, Some new results on odd perfect

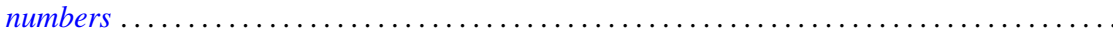

M. Edelstein and L. Keener, Characterizations of infinite-dimensional and nonreflexive

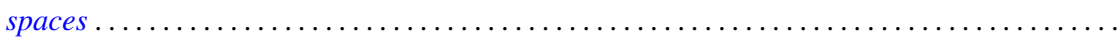

Francis James Flanigan, On Levi factors of derivation algebras and the radical embedding

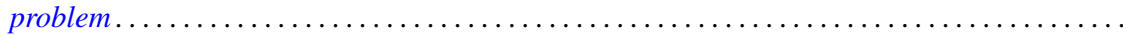

Harvey Friedman, Provable equality in primitive recursive arithmetic with and without

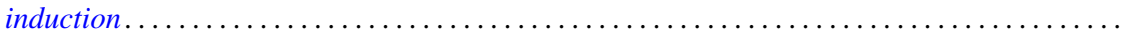

Joseph Braucher Fugate and Lee K. Mohler, The fixed point property for tree-like continua with

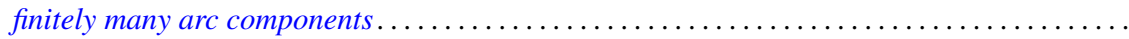

John Norman Ginsburg and Victor Harold Saks, Some applications of ultrafilters in

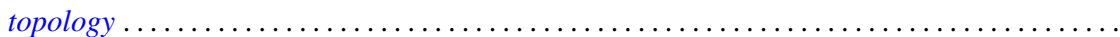

Arjun K. Gupta, Generalisation of a "square" functional equation .....................

Thomas Lee Hayden and Frank Jones Massey, Nonlinear holomorphic semigroups ..........

V. Kannan and Thekkedath Thrivikraman, Lattices of Hausdorff compactifications of a locally

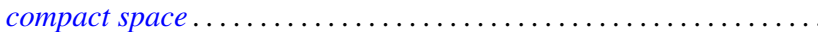

J. E. Kerlin and Wilfred Dennis Pepe, Norm decreasing homomorphisms between group

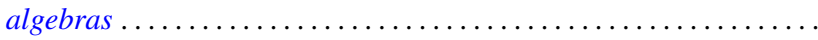

Young K. Kwon, Behavior of $\Phi$-bounded harmonic functions at the Wiener boundary ...

Richard Arthur Levaro, Projective quasi-coherent sheaves of modules .

Chung Lin, Rearranging Fourier transforms on groups...........................

David Lowell Lovelady, An asymptotic analysis of an odd order linear differential equation . . 4475

Jerry Malzan, On groups with a single involution .......................... 481

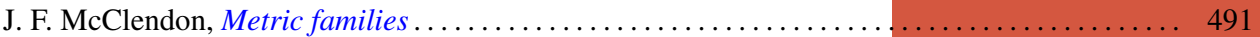

Carl Pomerance, On multiply perfect numbers with a special property .

Mohan S. Putcha and Adil Mohamed Yaqub, Polynomial constraints for finiteness of semisimple rings. .

Calvin R. Putnam, Hyponormal contractions and strong power convergence . . . . . . . . . 531

Douglas Conner Ravenel, Multiplicative operations in $\mathrm{BP} * \mathrm{BP} \ldots \ldots \ldots \ldots \ldots \ldots \ldots \ldots \ldots .539$

Judith Roitman, Attaining the spread at cardinals which are not strong limits . . . . . . . . . 545

Kazuyuki Saitô, Groups of *-automorphisms and invariant maps of von Neumann algebras . . . 553

Brian Kirkwood Schmidt, Homotopy invariance of contravariant functors acting on smooth

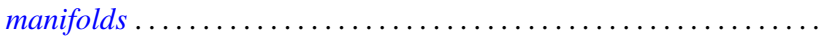

Kenneth Barry Stolarsky, The sum of the distances to $N$ points on a sphere.

Mark Lawrence Teply, Semiprime rings with the singular splitting property.

J. Pelham Thomas, Maximal connected Hausdorff spaces..............

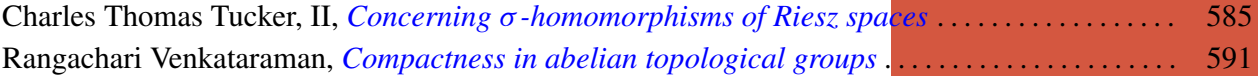

William Charles Waterhouse, Basically bounded functors and flat sheaves . . . . . . . . . . . 597

David Westreich, Bifurcation of operator equations with unbounded linearized part ......... 611

William Robin Zame, Extendibility, boundedness and sequential convergence in spaces of 